\title{
ON THE EFFICIENCY OF STORMWATER DETENTION TANKS IN POLLUTANT REMOVAL
}

\author{
A. RAIMONDI \& G. BECCIU \\ Politecnico di Milano, Italy.
}

\begin{abstract}
In the design of a stormwater detention tank is important to guarantee a sufficient retention time for the sedimentation of suspended solids, the biological uptake of nutrients and the die-off of bacteria carried in rainwaters. Long retention times increase the capacity of pollutant removal, but also the possibility of spills in downstream receivers and the risk of environmental pollution. In this paper, an analytical probabilistic approach, to estimate the probability distribution function of the average retention time and the efficiency in pollutant removal of stormwater tanks has been proposed. The possibility of water mixing from consecutive runoff events and storage carryover due to successive rainfall events has been considered. The method has been applied to a case study in Milano, Italy.

Keywords: analytical probabilistic approach, environmental pollution control, stormwater detention tanks.
\end{abstract}

\section{INTRODUCTION}

Detention tanks are often used in modern urban drainage systems to achieve both quantitative and qualitative control of stormwater runoff. The first goal is achieved by storing part of runoff to reduce overflows to receiving water bodies; the second by ensuring proper water retention times.

The two goals are in conflict with each other since the growth of retention time increases the probability of spills from the tank. A proper design should consider both these aspects, also trying to limit costs [1-4].

The key point is the definition of an optimal retention time. For simplicity, many governments suggest the use of a drawdown time (time to drain a full storage) in the range of 24-48 $\mathrm{h}$. This assumption has been supported by different studies that concluded that shorter retention times could be not sufficient to allow a good sedimentation of most of suspended solids, while longer retention times are useless because most of particles contained in stormwaters sediment in few days [5]. Moreover, long retention times can cause smell problems resulting from the combination of wastewater quality, temperature and time [6]. Other studies on retention time $[7,8]$, observed that it also depends on the size of particles and concluded that a retention time of $24 \mathrm{~h}$ can remove most of particles less than $10 \mu \mathrm{m}$ diameter and all the particles larger than $10 \mu \mathrm{m}$.

Although retention time is often regarded as a deterministic parameter [9-12], many authors have observed that it should be considered a random variable [13-15]. Therefore, also tank efficiency in pollution removal should be considered as a random variable.

In this paper, an analytical probabilistic approach is proposed, for the estimation of the probability distribution function of the average retention time to be used for the tank efficiency 
estimation. The goal is, starting from a more rigorous definition of average retention time consider the possibility of spill when the volume is full and the possibility of water mixing from consecutive rainfalls due to pre-filling of the storage from previous events.

Final expressions have been applied on a case study in Milano, Italy; the series of rainfall data recorded at Milano-Monviso gauge station in the period 1991-2005 has been used. Results from the proposed method have been compared with those obtained from the continuous simulation of observed data.

\section{TANK EFFICIENCY}

Efficiency of a detention tank in terms of pollutant removal can be defined as the fraction of inflow particles that are trapped inside. A particle is trapped when its retention time, defined as the time passed inside the tank before overflow or sedimentation, is greater than or equal to the time of sedimentation, defined as the time needed to reach the tank bottom.

If sedimentation is supposed to be mainly driven by gravity and interaction among particles is neglected, the vertical component of velocity $V_{s}$ for a particle of diameter $D$ is expressed by the Stokes equation:

$$
V_{s}(D)=\frac{g \cdot\left(\rho_{p}-\rho\right) \cdot D^{2}}{18 \cdot \mu}
$$

Where $\rho_{\mathrm{p}}$ and $\rho$ are the densities of particles and water and $\mu$ is the cinematic viscosity of water.

Considering the velocity expressed by eqn (1) as a mean value of a time variant physical quantity, the time required to a particle on the water surface to settle on the tank bottom $t_{s}$ is simply equal to the ratio:

where $H$ is the water depth.

$$
t_{s}(D)=\frac{H}{V_{s}(D)}
$$

Equating $t_{s}(D)$ to an assumed retention time $t_{r}$, the limit diameter $D_{\mathrm{o}}$ can be calculated as:

$$
D_{o}=\left[\frac{18 \cdot \mu \cdot H}{g \cdot\left(\rho_{p}-\rho\right) \cdot t_{r}}\right]^{1 / 2}
$$

All the particles with a diameter $D \geq D_{\mathrm{o}}$ have a sedimentation time smaller than or equal to the retention time $t_{r}$ and so are trapped. The fraction of these particles can be estimated by field sieve analysis or from literature data on sediments in stormwater runoff. The others can be trapped or not according to their distance from the tank bottom.

Assuming a uniform distribution along the water depth of particle number of each diameter, the fraction of particles $r_{s}$ with $D<D_{\mathrm{o}}$ that is trapped is equal to:

$$
r_{s}(D)=\frac{h_{m}(D)}{H}=\frac{t_{r} \cdot V_{s}(D)}{t_{r} \cdot V_{s}\left(D_{o}\right)}=\frac{V_{s}(D)}{V_{s}\left(D_{o}\right)}=\left(\frac{D}{D_{o}}\right)^{2}
$$

where $h_{m}$ is the distance from the tank bottom for which a particle with $D<D_{\mathrm{o}}$ has a sedimentation time equal to $t_{r}$.

Tank efficiency in particle removal can then be calculated by the following relationship:

$$
E=\left(1-F_{o}\right)+\frac{1}{D_{o}^{2}} \cdot \int_{0}^{D_{0}} D^{2} \cdot f_{D}(x) \cdot d x
$$


where $F_{o}$ is the fraction of particles with a diameter smaller than $D_{\mathrm{o}}$ and $f_{\mathrm{D}}(\mathrm{x})$ is the slope of the tangent of the particle gradation curve.

From the combination of eqns (3) and (5) results that the tank efficiency $E$ is a function of the retention time $t_{R}$. If inflow and outflow are equal and time invariant, as in steady flow sedimentation tanks, this time is constant and simply calculated by the relationship:

$$
t_{R}^{*}=\frac{B \cdot H \cdot L}{Q}=\frac{W_{o}}{Q}
$$

where $B$ and $L$ are the tank width and the length and $Q$ the constant flow.

Stormwater tanks, however, are characterized by variable inflow and outflow, causing a continuous process of filling and emptying. So, retention time is also variable and often an average value is considered, depending on inflow and outflow pattern.

Due to hydrologic processes of rainfall-runoff transformation acting on the upstream urban catchment, both this average retention time and the related tank efficiency can be regarded as random variables.

It has to be highlighted that using an average retention time implies also that the tank efficiency given by eqn (5) should be considered as an 'average' too.

In the next paragraph, the probability distribution of average retention time is derived, to be used together with eqn (5) to achieve a probabilistic estimation of this 'average' tank efficiency.

\section{PROBABILITY DISTRIBUTION FUNCTION OF AVERAGE RETENTION TIMES}

In the estimation of the probability distribution function of average retention times some assumptions for the simplification of the analytical probabilistic model have been made:

- On-line stormwater detention tank;

- Inflows have been considered of constant intensity (rectangular events);

- Constant outflows rate $Q_{O}(t)=q$;

- Runoff volume for unit of catchment surface $v$ has been assumed equal to rainfall depth $h$ less than an Initial Abstraction IA multiplied by the runoff coefficient $\phi$, that is $v=\varphi \cdot(h-I A)$;

- Rainfall-runoff transformation has been neglected, as typical for small catchments with short corrivation times. For highly urbanized catchment where $I A$ tends to zero and $\phi$ tends to one, runoff volume can be considered equal to rainfall volume, $v=h$ and runoff duration can be assumed equal to rainfall duration;

- Use of the Inter Event Time Definition IETD, to isolate independent rainfall events from the continuous chain of storms: if the dry time between two consecutive rainfall events is smaller than IETD, the two events have been joined together into a single event, otherwise they have been considered independent;

- Exponential distribution of the hydrological variables involved in the storage process (rainfall depth $h$ and duration $\theta$, interevent time $d$ ):

$$
\begin{gathered}
\mathrm{f}_{\mathrm{h}}=\xi \cdot \mathrm{e}^{-\xi \cdot \mathrm{h}} \\
\mathrm{f}=\lambda \cdot \mathrm{e}^{-\lambda \cdot \theta} \\
\mathrm{f}_{\mathrm{d}}=\psi \cdot \mathrm{e}^{-\psi \cdot(\mathrm{d}-\mathrm{IETD})}
\end{gathered}
$$


where $\xi=1 / \mu_{h}, \lambda=1 / \mu_{\theta}$ and $\psi=1 /\left(\mu_{d}-I E T D\right)$, with $\mu_{h}$ : average rainfall depth, $\mu_{\theta}$ : average rainfall duration, $\mu_{d}$ : average interevent time.

To estimate the probability distribution function of average retention times, care must be taken to the definition of retention time. If the hypothesis of plug flow (flow parcels leave the basin in the same order they entered) and completely mixed flow are considered, the average retention time. $\bar{t}_{R}$ can be calculated as the difference between the average release time $\bar{t}_{O}$ and the average input time $\bar{t}_{I} s$ that is the horizontal distance between centroids of inflow and outflow hydrographs:

$$
\bar{t}_{R}=\bar{t}_{O}-\bar{t}_{I}
$$

On the assumption of independence of inflow and outflow hydrograph, eqn (10) can be simplified as follow:

$$
\begin{aligned}
\bar{t}_{R} & =\bar{t}_{O}-\bar{t}_{I}=\int_{0}^{t_{O}} \tau_{O} \cdot f_{\tau_{O}}\left(\tau_{O}\right) \cdot d \tau_{O}-\int_{0}^{t_{I}} \tau_{I} \cdot f_{\tau_{I}}\left(\tau_{I}\right) \cdot d \tau_{I}= \\
& =\frac{1}{V_{O}} \cdot \int_{0}^{t_{0}} \tau \cdot Q_{O}(\tau) \cdot d \tau-\frac{1}{V_{I}} \cdot \int_{0}^{t_{I}} \tau \cdot Q_{I}(\tau) \cdot d \tau
\end{aligned}
$$

$t_{O}$ : release time, that coincides with emptying time;

$t_{I}$ : input time;

$V_{O}$ : outflow volume;

$V_{I}$ : inflow volume;

$Q_{O}$ : outflow rate;

$Q_{I}$ : inflow rate.

For constant outflow rate, $Q_{O}(\tau)=q=$ const., the average release time results:

$$
\overline{t_{O}}=\frac{1}{V_{O}} \cdot \int_{0}^{t_{O}} \tau \cdot Q_{O}(\tau) \cdot d \tau=\frac{\frac{1}{2} \cdot t_{O}^{2} \cdot q}{t_{O} \cdot q}=\frac{1}{2} \cdot t_{O}
$$

If even inflow rate is constant during the event, $Q_{I}(\tau)=q_{I}=$ const., the average inflow time results:

$$
\overline{t_{I}}=\frac{1}{V_{I}} \cdot \int_{0}^{t_{I}} \tau \cdot Q_{I}(\tau) \cdot d \tau=\frac{\frac{1}{2} \cdot t_{I}^{2} \cdot q_{I}}{t_{I} \cdot q_{I}}=\frac{1}{2} \cdot t_{I}
$$

Considering the simplifying hypothesis of eqns (11)-(13) becomes:

$$
\bar{t}_{R}=\frac{1}{2} \cdot\left(t_{O}-t_{I}\right)=\frac{1}{2} \cdot t_{O} \cdot\left(1-\frac{t_{I}}{t_{O}}\right)=\frac{1}{2} \cdot t_{O} \cdot\left(1-\frac{q}{q_{I}}\right)
$$

Sometimes, the tank is not completely empty when a new runoff event occurs, that is there is a carryover from previous runoffs. Obviously, the probability of pre-filling increases when outflow rate is low, as in the case of tanks for the enhancement of water quality (e.g. first flush tanks). For this reason, in the estimation of the probability distribution function of average 
retention times the possibility of water mixing from two consecutive runoffs has been considered.

In the following, specific variable (for unit of area) have been used. For a couple of consecutive runoffs $i$ and $i+1$, if $w$ is the storage volume and $q$ the constant outflow rate, two different conditions can occur:

- $\quad w / q \leq I E T D$ : the possibility of pre-filling of the storage volume from the event $i$ at the beginning of the event $i+1$ is excluded;

- $\quad w / q>I E T D$ : the storage volume could be not completely empty from the event $i$ at the beginning of the event $i+1$ and pre-filling could occur.

In case there is no water carryover from event $i$ storage volume is completely empty at the beginning of event $i+1\left(t_{O}<\theta_{i}+d_{i}\right)$, as shown in Fig. 1 .

If the active volume is partially filled at the beginning of event $i+l$ (Fig. 2), water mixing from two consecutive events has to be considered in the derivation of the probability distribution function of average retention times.

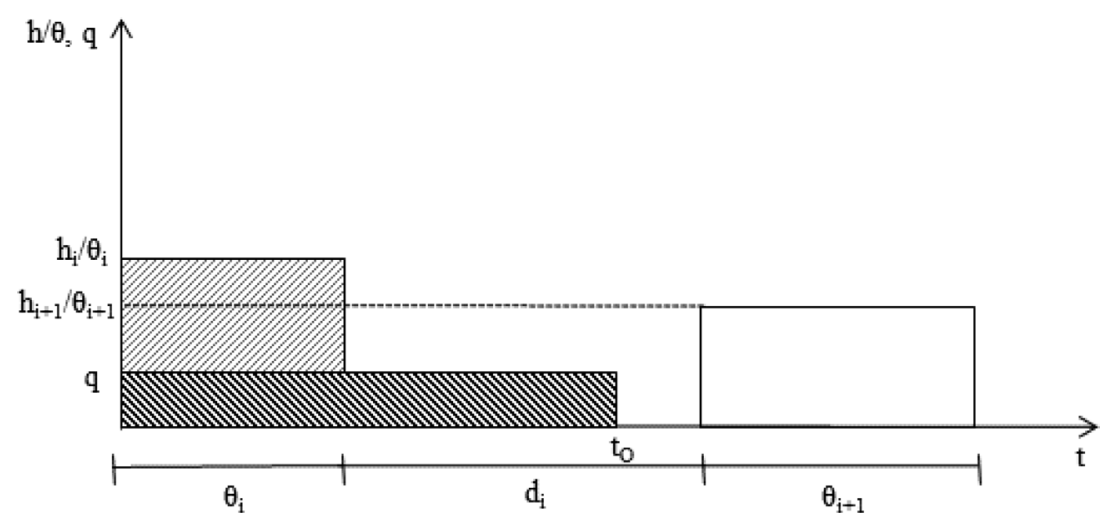

Figure 1: Couple of runoffs without pre-filling.

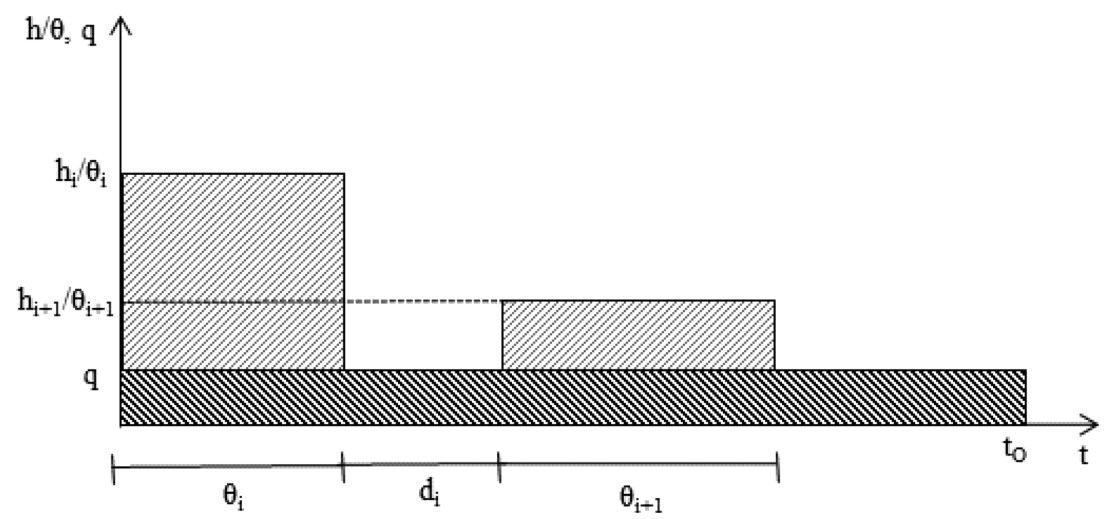

Figure 2: Couple of runoffs with pre-filling. 
Generally inflow rates can be higher or lower than outflow rates; in the first case, the possibility of spills if storage volume is full at the end of each event has been considered.

3.1 Condition w/q $\leq$ IETD: pre-filling is excluded

In this case, inter-event time is always enough to have no prefilling and events are independent. The inflow time $t_{I}$ coincides with the runoff duration $\theta$, so that eqn (14) becomes:

$$
\bar{t}_{R}=\frac{1}{2} \cdot\left(t_{O}-\theta\right)
$$

The emptying time $t_{O}$ can be expressed by:

$$
t_{O}=\left\{\begin{array}{cc}
0 & h_{i}-q \cdot \theta_{i} \leq 0 \\
h_{i} / q & 0<h_{i}-q \cdot \theta_{i}<w \\
\theta_{i}+w / q & h_{i}-q \cdot \theta_{i} \geq w
\end{array}\right.
$$

Substituting eqn (11) in eqn (10), the average retention time results:

$$
\bar{t}_{R}=\frac{1}{2} \cdot\left\{\begin{array}{cc}
0 & h_{i}-q \cdot \theta_{i} \leq 0 \\
h_{i} / q-\theta_{i} & 0<h_{i}-q \cdot \theta_{i}<w \\
w / q & h_{i}-q \cdot \theta_{i} \geq w
\end{array}\right.
$$

It has to be observed that the average retention time has an upper limit, equal to (w/q)/2, and its probability distribution function is truncated in the upper tail. From eqns (17), the probability that the average retention time is greater than a fixed value $t_{x}$ is then expressed as:

$$
\begin{aligned}
F_{\bar{t}_{R}} & =P\left(t_{x}<\bar{t}_{R}\right)=P\left(t_{x}<\bar{t}_{R}<\frac{w}{2 \cdot q}\right)+P\left(\bar{t}_{R}=\frac{w}{2 \cdot q}\right) \\
& =\int_{\theta=0}^{\infty} f_{\theta} \cdot d \theta \cdot \int_{h=q \cdot\left(\theta+2 \cdot t_{x}\right)}^{w+q \theta} f_{h} \cdot d h+\int_{\theta=0}^{\infty} f_{\theta} \cdot d \theta \cdot \int_{h=w+q \theta}^{\infty} f_{h} \cdot d h=\frac{e^{-2 \xi q t_{x}}}{1+q^{*}}
\end{aligned}
$$

and $\mathrm{q}^{*}=\xi \cdot \mathrm{q} / \lambda$.

\subsection{Condition w/q >IETD: possibility of pre-filling}

In case of pre-filling from event $i$ at the beginning of event $i+1$, the average input time $\bar{t}_{I}$ can be expressed by:

$$
\begin{aligned}
\bar{t}_{I} & =\frac{1}{V_{I}} \cdot \int_{0}^{\tau_{I}} \tau \cdot Q_{I}(\tau) \cdot d \tau=\frac{1}{V_{I}} \cdot\left[Q_{I, i} \int_{0}^{\theta_{i}} \tau \cdot d \tau+Q_{I, i+1} \int_{\theta_{i}+d_{i}}^{\theta_{i}+d_{i}+\theta_{i+1}} \tau \cdot d \tau\right]= \\
& =\frac{h_{i} \cdot \theta_{i} / 2+h_{i+1} \cdot\left(\theta_{i}+d_{i}+\theta_{i+1} / 2\right)}{h_{i}+h_{i+1}}
\end{aligned}
$$


Equation (11) in this case results:

$$
\bar{t}_{R}=\bar{t}_{O}-\bar{t}_{I}=\frac{1}{2} \cdot\left[t_{O}-\frac{h_{i} \cdot \theta_{i}+h_{i+1} \cdot\left(2 \cdot \theta_{i}+2 \cdot d_{i}+\theta_{i+1}\right)}{h_{i}+h_{i+1}}\right]
$$

The emptying time $t_{O}$ can be expressed by:

$$
t_{O}=\left\{\begin{array}{cc}
0 & \text { case I } \\
h_{i} / q & \text { cases } I I-I I I-I V \\
w / q+\theta_{i} & \text { cases } V-V I I \\
w / q+\theta_{i+1} & \text { caseVI } \\
\left(h_{i}+h_{i+1}\right) / q & \text { case VIII } \\
\theta_{i}+d_{i}+\theta_{i+1}+w / q & \text { cases } I X-X \\
\theta_{i}+\left(w+h_{i+1}\right) / q & \text { case XI }
\end{array}\right.
$$

case I - Rainfall intensity lower than outflow rate:

$$
h_{i}-q \cdot \theta_{i} \leq 0 ; h_{i+1}-q \cdot \theta_{i+1} \leq 0
$$

case II - No pre-filling, event $i$ without spills:

$$
0<h_{i}-q \cdot \theta_{i} \leq w ; h_{i}-q \cdot \theta_{i}-q \cdot d_{i} \leq 0 ;
$$

case III - Intensity of event $i$ lower than outflow rate, event $i+1$ without spills:

$$
h_{i}-q \cdot \theta_{i} \leq 0 ; 0<h_{i+1}-q \cdot \theta_{i+1} \leq w ;
$$

case $I V$ - Pre-filling, event $i$ without spills, event $i+1$ with intensity lower than outflow rate:

$$
0<h_{i}-q \cdot \theta_{i} \leq w ; h_{i}-q \cdot \theta_{i}-q \cdot d_{i}>0 ; h_{i}-q \cdot \theta_{i}-q \cdot d_{i}+h_{i+1}-q \cdot \theta_{i+1} \leq 0 ;
$$

case $V$ - No pre-filling, event $i$ with spills:

$$
h_{i}-q \cdot \theta_{i}>w ; w-q \cdot d_{i} \leq 0
$$

case VI - Event $i$ with intensity lower than outflow rate, event $i+1$ with spills:

$$
h_{i}-q \cdot \theta_{i} \leq 0 ; h_{i+1}-q \cdot \theta_{i+1}>w
$$

case VII - Pre-filling, event $i$ with spills, event $i+1$ with intensity lower than outflow rate:

$$
h_{i}-q \cdot \theta_{i}>w ; w-q \cdot d_{i}>0 ; w-q \cdot d_{i}+h_{i+1}-q \cdot \theta_{i+1} \leq 0 ;
$$

case VIII - Pre-filling, both event $i$ and event $i+1$ without spills:

$$
0<h_{i}-q \cdot \theta_{i} \leq w ; h_{i}-q \cdot \theta_{i}-q \cdot d_{i}>0 ; 0<h_{i}-q \cdot \theta_{i}-q \cdot d_{i}+h_{i+1}-q \cdot \theta_{i+1} \leq w ;
$$

case IX-Pre-filling, both and event $i$ and event $i+l$ with spills:

$$
h_{i}-q \cdot \theta_{i}>w ; w-q \cdot d_{i}>0 ; w-q \cdot d_{i}+h_{i+1}-q \cdot \theta_{i+1}>w ;
$$

case $X-$ Pre-filling, event $i$ without spills, event $i+l$ with spills: 


$$
0<h_{i}-q \cdot \theta_{i} \leq w ; h_{i}-q \cdot \theta_{i}-q \cdot d_{i}>0 ; h_{i}-q \cdot \theta_{i}-q \cdot d_{i}+h_{i+1}-q \cdot \theta_{i+1}>w ;
$$

case XI - Pre-filling, event $i$ with spills and event $i+1$ without spills:

$$
h_{i}-q \cdot \theta_{i}>w ; w-q \cdot d_{i}>0 ; 0<w-q \cdot d_{i}+h_{i+1}-q \cdot \theta_{i+1} \leq w .
$$

For the same assumption on the probability distribution functions of rainfall depth, duration and interevent time considered above, that is $f_{h, i}=f_{h, i+i}=f_{h}, f_{\theta, i}=f_{\theta, i+1}=f_{\theta}$ and $f_{d}=f_{d, i}=f_{d, i+1}$, case III, case IV, case VI, case VII and case XI cannot occur and eqn (20) becomes:

$$
\overline{t_{R}}=\bar{t}_{O}-\bar{t}_{I}=\frac{1}{2} \cdot\left[t_{O}-2 \cdot \theta-d\right]
$$

Substituting eqn (21) in eqns (15) and (22), respectively, if a single event or a couple of chained events is considered, the average retention time results:

$$
\bar{t}_{R}=\left\{\begin{array}{cc}
0 & \text { case I } \\
0.5 \cdot(h / q-\theta) & \text { case II } \\
h / q-\theta-d / 2 & \text { caseVIII } \\
w /(2 \cdot q) & \text { cases } V-I X-X
\end{array}\right.
$$

Equation (23) is valid for $w / q>I E T D$ and for $t_{x}<w /(2 \cdot q)$.

The probability distribution function of average retention times results:

$$
\begin{gathered}
F_{\bar{t}_{R}}=P\left(t_{x}<\bar{t}_{R}\right)=P\left(t_{x}<\bar{t}_{R}<\frac{w}{2 \cdot q}\right)+P\left(\bar{t}_{R}=\frac{w}{2 \cdot q}\right) \\
=\int_{\theta_{1}}^{\theta_{2}} f_{\theta} \cdot d \theta \cdot \int_{d_{1}}^{d_{2}} f_{d} \cdot d d \cdot \int_{h_{1}}^{h_{2}} f_{h} \cdot d h+\int_{\theta_{3}}^{\theta_{4}} f_{\theta} \cdot d \theta \cdot \int_{d_{3}}^{d_{4}} f_{d} \cdot d d \cdot \int_{h_{3}}^{h_{4}} f_{h} \cdot d h \\
+\int_{\theta_{5}}^{\theta_{6}} f_{\theta} \cdot d \theta \cdot \int_{d_{6}}^{d_{5}} f_{d} \cdot d d \cdot \int_{h_{5}}^{h_{6}} f_{h} \cdot d h+\int_{\theta_{7}}^{\theta_{8}} f_{\theta} \cdot d \theta \cdot \int_{d_{7}}^{d_{8}} f_{d} \cdot d d \cdot \int_{h_{7}}^{h_{h}} f_{h} \cdot d h \\
\theta_{1}=\theta_{3}=\theta_{5}=\theta_{7}=0 ; \theta_{2}=\theta_{4}=\theta_{6}=\theta_{8}=\infty ; \\
d_{1}=d_{8}=2 \cdot t_{x} ; d_{2}=d_{4}=d_{5}=w / q ; d_{3}=d_{7}=I E T D ; d_{6}=\infty ; \\
h_{1}=h_{5}=q \cdot\left(\theta+2 \cdot t_{x}\right) ; h_{2}=h_{3}=h_{8}=q \cdot \theta+(q \cdot d+w) / 2 ; h_{4}=h_{6}=\infty ; h_{7}=q \cdot\left(t_{x}+d / 2+\theta\right) ;
\end{gathered}
$$

which solution is:

$$
F_{\bar{t}_{R}}\left(t_{x}\right)=\frac{1}{1+q^{*}} \cdot\left[(1-\beta) \cdot e^{\psi / \cdot I E T D-2 \cdot t_{x} \cdot(\psi+\xi \cdot q)}+\beta \cdot e^{-\xi \cdot q \cdot\left(\frac{I E T D}{2}+t_{x}\right)}\right]
$$

with: $\beta=2 \cdot \psi /(\mathrm{q} \cdot \xi+2 \cdot \psi)$ and $q^{*}=q \cdot \xi / \lambda$.

\section{CASE STUDY}

To test the reliability of derived expressions for the estimation of the probability distributions of average retention times, a case study in Milano, Italy, has been analyzed. The series of rainfall data recorded at Milano-Monviso gauge station in the period 1991-2005 has been used and IETD=10 hours has been assumed. 
Table 1: Main characteristics of rainfall variables.

\begin{tabular}{lrrrrr}
\hline $\boldsymbol{\mu}_{\mathrm{h}}[\mathrm{mm}]$ & 18.49 & $\mathrm{~V}_{\mathrm{h}}[-]$ & 1.15 & $\rho_{\mathrm{h}, \theta}[-]$ & 0.62 \\
$\boldsymbol{\mu}_{\theta}[\mathrm{h}]$ & 14.37 & $\mathrm{~V}_{\theta}[-]$ & 1.03 & $\rho_{\theta, d}[-]$ & 0.11 \\
$\boldsymbol{\mu}_{\mathrm{d}}[\mathrm{h}]$ & 172.81 & $\mathrm{~V}_{\mathrm{d}}[-]$ & 1.30 & $\rho_{\mathrm{d}, \mathrm{h}}[-]$ & 0.11 \\
\hline
\end{tabular}

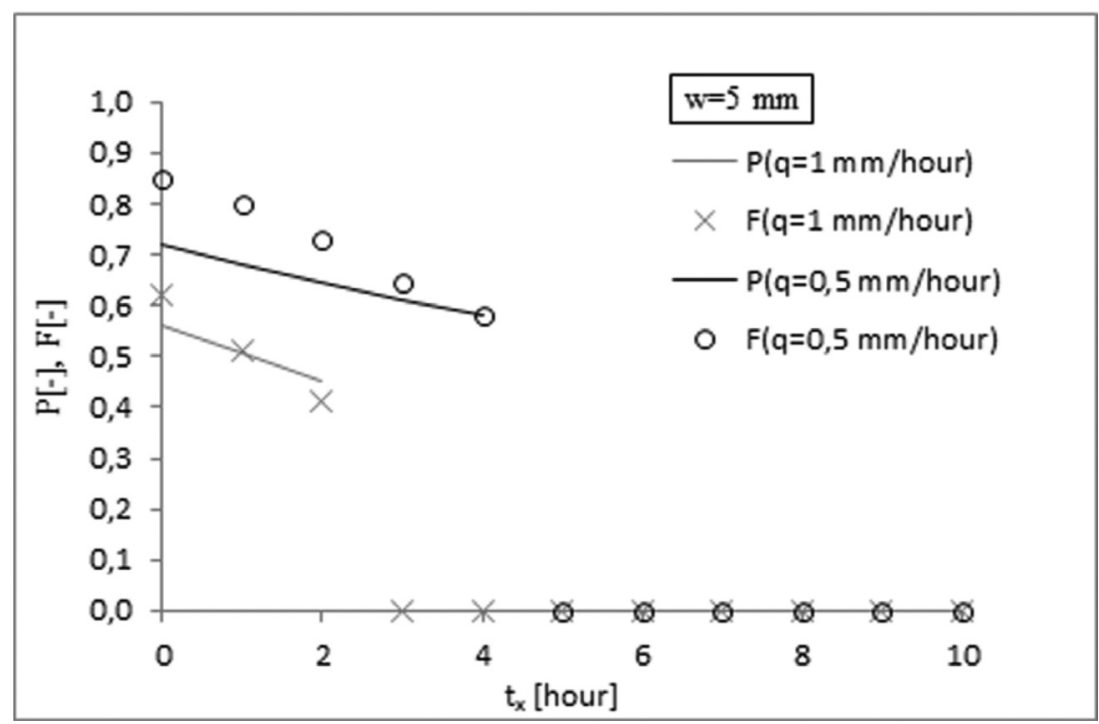

Figure 3: Probability and frequency distributions of average retention times $(\mathrm{w}=5 \mathrm{~mm}$; $\mathrm{q}=1 \mathrm{~mm} / \mathrm{h} ; \mathrm{q}=0.5 \mathrm{~mm} / \mathrm{h}$ ).

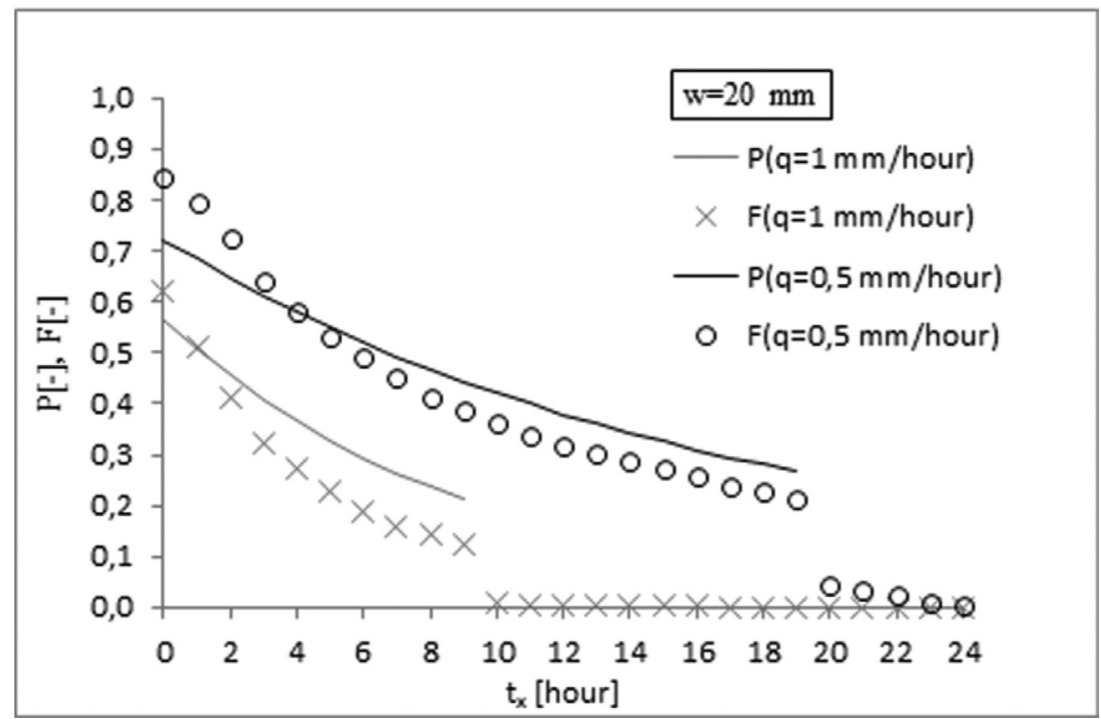

Figure 4: Probability and frequency distributions of average retention times $(\mathrm{w}=20 \mathrm{~mm}$; $\mathrm{q}=1 \mathrm{~mm} / \mathrm{h} ; \mathrm{q}=0.5 \mathrm{~mm} / \mathrm{h})$. 
The main characteristics (mean, variation coefficient and correlation index) of rainfall variables involved in storage process (rainfall depth $h$, rainfall duration $\theta$ and interevent time d) have been shown in Table 1 .

Outflow rates of $\mathrm{q}=0.5 \mathrm{~mm} / \mathrm{h}$ and $\mathrm{q}=1.0 \mathrm{~mm} / \mathrm{h}$ and a storage volume $\mathrm{w}=5 \mathrm{~mm}$ and $\mathrm{w}=$ $20 \mathrm{~mm}$ have been considered.

Figures 3 and 4 compare the probability distribution functions of the average retention time, calculated by eqns (18) and $(24 *)$ with the frequency distribution of simulated data, respectively, for $\mathrm{w}=5 \mathrm{~mm}$ and $\mathrm{w}=20 \mathrm{~mm}$ (continuous black line and circles for $\mathrm{q}=0.1$ $\mathrm{mm} / \mathrm{h}$ and continuous grey line and crosses for $\mathrm{q}=1.0 \mathrm{~mm} / \mathrm{h}$ ).

Differences in results can be due to:

- The simplifying assumption on the independence of input rainfall variables $h, \theta, d$ while in particular the correlation index between rainfall depth and rainfall duration is not negligible (Table 1);

- The simplifying assumption on exponential distribution of input rainfall variables $h, \theta$, $d$; as it can be deduced from Table 1, only the frequency distribution of rainfall durations perfectly fits an exponential probability distribution function $\left(\mathrm{V}_{\theta} \approx 1\right)$;

- The simplifying assumption of considering only a couple of consecutive event at time; if the outflow rate tends to zero, the number of chained events increases:

- The simplifying assumption of considering the probability distribution functions of rainfall characteristics of event $i$ equal to those of event $i+1\left(f_{h, i}=f_{h, i+i}=f_{h}, f_{\theta, i}=f_{\theta, i+1}=f_{\theta}\right.$ and $f_{d}=f_{d, i}=f_{d, i+1}$ ), that excludes cases III-IV-VI-VII-XI of eqn (21) in the resulting formula $(24 *)$

\section{CONCLUSIONS}

Proposed approach relates the efficiency of a stormwater detention tanks in pollutant removal with the retention time. In particular, the probability distribution function of the average retention time has been estimated. Derived formulas are easy to implement and can be a valid aid to engineer, when there are no long-term registration of records data and only the mean values of rainfall characteristics are available. Moreover, they can be used to size stormwater detention tanks because allow to analyze the influence of outflow rates and storage volumes on the probability distribution of the average retention time, that is on probability distribution of the efficiency of the storage in pollutant removal.

\section{REFERENCES}

[1] Carleton, J.N., Grizzard, T.J., Godrej, A.N. \& Post, H.E., Factors affecting the performance of stormwater treatment wetlands. Water Research, 35, pp. 1552-1562, 2001. http://dx.doi.org/10.1016/S0043-1354(00)00416-4

[2] Guo, Y. \& Adams, B.J., Analysis of detention ponds for storm water quality control. Water Resources Research, 35, pp. 2447-2456, 1999. http://dx.doi.org/10.1029/1999WR900124

[3] Becciu, G. \& Raimondi, A., Probabilistic analysis of spills from stormwater detention facilities, WIT Transactions on the Built Environment, 139, Urban Water II (ISSN: 1743-3509), WIT Press, 2014. http://dx.doi.org/10.2495/UW140141 
[4] Becciu, G. \& Raimondi, A., Probabilistic modeling of the efficiency of a stormwater detention facility. International Journal of Sustainable Development and Planning, pp. 1-11, DOI: 10.2495/SDP-V0-N0-1-11, 2015b.

[5] Walker, D.J., Modelling residence time in stormwater ponds. Ecological Engineering, 10, pp. 247-262, 1998.

http://dx.doi.org/10.1016/S0925-8574(98)00016-0

[6] Grundfos Wastewater, Design of Stormwater Tank: recommendations and layout, 2011.

[7] Li, Y., Lau, S., Kayhanian, M. \& Stenstrom, M.K., Particle size distribution in highway runoff. Journal of Environment Engineering, 13, pp. 1267-1276, 2005. http://dx.doi.org/10.1061/(ASCE)0733-9372(2005)131:9(1267)

[8] Li, Y., Lau, S., Kayhanian, M. \& Stenstrom, M.K., Dynamic characteristics of particle size distribution in highway runoff: implications for settling tank design. Journal of Environment Engineering, 132, pp. 852-861, 2006. http://dx.doi.org/10.1061/(ASCE)0733-9372(2006)132:8(852)

[9] Nix, S.J., Residence time in stormwater detention basins. Journal of Environment Engineering, 111, pp. 95-100, 1985. http://dx.doi.org/10.1061/(ASCE)0733-9372(1985)111:1(95)

[10] Werner, T.M. \& Kadlec, R.H., Application of residence time distributions to stormwater treatment systems. Ecological Engineering, 7, pp. 213-234, 1996. http://dx.doi.org/10.1016/0925-8574(96)00013-4

[11] Werner, T.M. \& Kadlec, R.H., Wetland residence time distribution modelling. Ecological Engineering, 15, pp. 77-90, 2000. http://dx.doi.org/10.1016/S0925-8574(99)00036-1

[12] Holland, J.F., Martin, J.F., Granata, T., Bouchard, V., Quigley, M. \& Brown, L., Effects of wetland depth and flow rate on residence time distribution characteristics. Ecological Engineering, 23, pp. 189-203, 2004. http://dx.doi.org/10.1016/j.ecoleng.2004.09.003

[13] Loganathan, G.V., Watkins, E.W. \& Kibler, D.F., Sizing storm-water detention basins for pollutant removal. Journal of Environment Engineering, 120, pp. 1380-1399, 1994. http://dx.doi.org/10.1061/(ASCE)0733-9372(1994)120:6(1380)

[14] Papa, F., Adams, B.J. \& Guo, Y., Detention time selection for stormwater quality control ponds. Canadian Journal of Civil Engineering, 26, pp. 72-82, 1999. http://dx.doi.org/10.1139/198-046

[15] Becciu, G. \& Raimondi, A., Probabilistic analysis of the retention time in stormwater detention facilities. Procedia Engineering, pp. 1299-1307, 2015a. http://dx.doi.org/10.1016/j.proeng.2015.08.951

[16] Raimondi, A. \& Becciu, G., On pre-filling probability of flood control detention facilities. Urban Water Journal, 12, pp. 344-351, 2015. http://dx.doi.org/10.1080/1573062X.2014.901398 